sind die Auswirkungen, die sich ergeben, wenn Moleküle in bestimmten Stellungen relativ zum elektrischen Feld - die sie etwa auch im Laufe einer freien Bewegung im Feld einnehmen können - bevorzugt dissoziieren, evtl. auch ionisiert werden.
Der Deutschen Forschungsgemeinschaft und dem Verband der Chemischen Industrie danken wir für die großzügige Förderung der vorliegenden Arbeit durch Sachbeihilfen.

\title{
Eine erweiterte Intensitätsfunktion für Elektronenbeugung an Gasen und ihre Interpretation durch kinematische Asymmetrien 1. Mitteilung
}

\author{
Werner Freyland, Joachim Haase und Werner Zeil \\ Lehrstuhl für Chemische Physik der Universität Kiel * \\ (Z. Naturforschg. 21 a, 1945-1952 [1966] ; eingegangen am 22. Juni 1966)
}

\begin{abstract}
Es wurden eine erweiterte Intensitätsfunktion und eine erweiterte Radialverteilungsfunktion für Elektronenbeugung an Gasen berechnet. Die Bestimmung der Atombewegung in einem Molekül mit Hilfe der Elektronenbeugung wird in diesem Zusammenhang von neuen Gesichtspunkten aus diskutiert. Eine aus den Erweiterungen resultierende Korrektur für die gemessenen Atomabstände wird angegeben.
\end{abstract}

In Anlehnung an eine Arbeit von $\mathrm{J}_{\mathrm{AmES}}{ }^{1}$ haben J. Karle und I. Karle ${ }^{2}$ den Einfluß von Atomschwingungen in Molekülen auf die Streuintensität von an Gasen gestreuten Elektronen untersucht. In ihren Rechnungen haben die letztgenannten Autoren die explizite Reihenentwicklung des Abstandes eines Atompaares im Molekül nach dem linearen Term abgebrochen und den quadratischen Term auf Grund einer mit Fehlern behafteten Abschätzung vernachlässigt. Nachdem in letzter Zeit die Strukturbestimmung von Molekülen mit Hilfe der Elektronenbeugung vom Experiment her um einiges verbessert worden ist (siehe z. B. ${ }^{3}$ ), schien es uns interessant zu wissen, von welcher Größenordnung die in ${ }^{2}$ gemachten Vernachlässigungen sind. Bei diesen Untersuchungen, die analog den in ${ }^{1}$ und ${ }^{2}$ beschriebenen Lösungswegen durchgeführt wurden **, und vor allem bei der Interpretation der Resultate stießen wir auf folgenden Sachverhalt.

Der maßgebliche Strukturparameter im Streuexperiment ist der Betrag des Abstandes, um den zwei Atome in jedem Augenblick voneinander entfernt sind. Danach ist es notwendig, den "wirkli-

* Derzeitige Anschrift: (75) Karlsruhe, Hertzstr. 16, Bau 35 (Westhochschule).

** Bei James und Karle-Karle ist die Anzahl der Schwingungsfreiheitsgrade mit $3 N$ angesetzt worden, wobei $N$ die Anzahl der Atome im Molekül bezeichnet. Wir haben im folgenden mit $L$ Freiheitsgraden gerechnet, worin $L=3 N-6$ für nichtlineare Moleküle und $L=3 N-5$ für lineare Moleküle ist. chen Bewegungsverlauf" der Atome und die damit verbundenen Abstandsänderungen möglichst genau zu kennen. Dies erfordert aber auch, daß man $\mathrm{Ab}$ standsänderungen, die auf Grund der Kinematik im Molekül unsymmetrisch bezüglich des Gleichgewichtsabstandes $r_{\mathrm{e}}$ sind, obwohl der harmonische Potentialansatz beibehalten wird, bei der Auswertung der Streuexperimente berücksichtigt. Umgekehrt wird in dieser Arbeit gezeigt, daß man diese Berücksichtigung durch Erweiterung der bisher verwendeten Intensitätsfunktion erhalten kann.

\section{Theorie}

Die Streuintensität für ein Paar $(i, j)$ von schwingenden Atomen ist gegeben durch ${ }^{4}$ :

$$
I_{i j}(s)=\text { const } \cdot f_{i} \cdot f_{j} \int_{0}^{\infty} P\left(r_{i j}\right) \frac{\sin \left(s r_{i j}\right)}{s r_{i j}} \mathrm{~d} r_{i j} .
$$

Darin bedeuten $f_{i}$ und $f_{j}$ die Streuamplituden des $i$-ten und $j$-ten Atoms, $r_{i j}$ den Abstand des $i$-ten vom $j$-ten Atom, $s=(4 \pi / \lambda) \cdot \sin (\vartheta / 2)$; die Konstante hat

1 R. W. JAMEs, Phys. Z. 33, 737 [1932].

2 J. Karle u. I. L. KarLe, J. Chem. Phys. 18, 957 [1950].

3 W. ZeiL, J. Hase u. L. WegmanN, Z. Instrumentenk. 74, 84 [1966].

4 P. Debye, J. Chem. Phys. 9, 55 [1941]. 
den Wert const $=\left[\left(e^{2} / E\right) / R^{2}\right] \cdot I_{0} \quad(e=$ Ladung des Elektrons, $E=$ kinetische Energie des einfallenden Elektrons, $I_{0}=$ einfallende Streuintensität, $R=$ Beugungsabstand). $P\left(r_{i j}\right) \mathrm{d} r_{i j}$ ist die Wahrscheinlichkeit, daß der Abstand zwischen Atom $i$ und $j$ zwischen $r_{i j}$ und $r_{i j}+\mathrm{d} r_{i j}$ liegt. Nach BLocH ${ }^{5}$ ist diese Wahrscheinlichkeitsfunktion für den harmonischen Oszillator gegeben durch:

mit

$$
\begin{gathered}
W\left(r_{i j}, T\right)=\left(\frac{4 \pi v}{h} \tanh \frac{h v}{2 k T}\right)^{1 / 2} \exp \left(-\frac{4 \pi^{2} v}{h} r_{i j}^{2} \tanh \frac{h v}{2 k T}\right), \\
\left(\frac{4 \pi v}{h} \tanh \frac{h v}{2 k T}\right)^{1 / 2}=\left(\frac{B}{\pi}\right)^{1 / 2} \text { und } \int_{-\infty}^{+\infty} W\left(r_{i j}\right) \mathrm{d} r_{i j}=1 ;
\end{gathered}
$$

$v$ ist die klassische Eigenfrequenz des Oszillators.

Obwohl diese Beziehungen nur für einen einzelnen Oszillator (2-atomiges Molekül) gelten, lassen sie sich mit Hilfe der Theorie der Normalschwingungen auf vielatomige Moleküle übertragen.

Um nun (1) weiter auswerten zu können, verfolgen wir zunächst den Weg von KarLE und KARLE ${ }^{2}$ und schreiben in einem kartesischen Koordinatensystem $\xi, \eta, \zeta$ den Abstand $r_{i j}$ als Funktion seiner Gleichgewichtskomponenten $\xi_{i j}=\xi_{i}-\xi_{j}$, usw.; die Auslenkung des $i$-ten Atoms wird durch die Verschiebungskoordinaten $\xi^{i}, \eta^{i}, \zeta^{i}$ beschrieben. Die Abweichungen vom Gleichgewicht sind dann $\xi^{i j}=\xi^{i}-\xi^{j}$, usw. Damit wird

$$
r_{i j}=\left[\left(\xi_{i j}+\xi^{i j}\right)^{2}+\left(\eta_{i j}+\eta^{i j}\right)^{2}+\left(\zeta_{i j}+\zeta^{i j}\right)^{2}\right]^{1 / 2} .
$$

Nach Entwicklung der Wurzel unter Vernachlässigung von $\left(\xi^{i j}\right)^{2}$ usw. ergibt sich

$$
r_{i j}={ }^{(\mathrm{e})} r_{i j}+\frac{1}{{ }^{(\mathrm{e})} r_{i j}}\left(\xi_{i j} \xi^{i j}+\eta_{i j} \eta^{i j}+\zeta_{i j} \zeta^{i j}\right)-\frac{1}{2^{\left({ }^{(e)}\right.} r_{i j}{ }^{3}}\left(\xi_{i j} \xi^{i j}+\eta_{i j} \eta^{i j}+\zeta_{i j} \zeta^{i j}\right)^{2}+\ldots
$$

KARLE hat den dritten Term der Entwicklung auf Grund einer Abschätzung mit fehlerhaft ausgewerteten Integralen - was wir weiter unten zeigen werden - vernachlässigt. Wir haben dieses Glied für die weitere Auswertung von (1) mitgenommen. Analog der Arbeit von $\mathrm{JAMES}^{1}$ transformieren wir die $\xi^{i}, \eta^{i}, \zeta^{i}$ auf Normalkoordinaten $Q_{n}$ :

$$
\xi^{i}=\sum_{n=1}^{L} \alpha_{n}{ }^{i} Q_{n}, \quad \eta^{i}=\sum_{n=1}^{L} \beta_{n}{ }^{i} Q_{n}, \quad \zeta^{i}=\sum_{n=1}^{L} \gamma_{n}{ }^{i} Q_{n} .
$$

Damit können wir (5) in den $Q_{n}$ schreiben

mit

$$
\begin{aligned}
& r_{i j}={ }^{(\mathrm{e})} r_{i j}+\sum_{n=1}^{L} c_{n} Q_{n}+\sum_{m, n=1}^{L} k_{m n} Q_{m} Q_{n} \\
& c_{n}=\frac{1}{{ }^{(\mathrm{e})} r_{i j}}\left[\xi_{i j}\left(\alpha_{n}^{i}-\alpha_{n}{ }^{j}\right)+\eta_{i j}\left(\beta_{n}{ }^{i}-\beta_{n}{ }^{j}\right)+\zeta_{i j}\left(\gamma_{n}{ }^{i}-\gamma_{n}{ }^{j}\right)\right] .
\end{aligned}
$$

Durch Vergleich von (5) und (7) findet man für die $k_{m n}$

In den $Q_{n}$ erhält (2) die Form

$$
k_{m n}=-\frac{1}{2^{\left({ }^{(e)}\right.} r_{i j}} c_{n} \cdot c_{m} \text {. }
$$

$$
W\left(Q_{1}, \ldots, Q_{L}\right)=\prod_{n=1}^{L}\left(\frac{B_{n}}{\pi}\right)^{1 / 2} \exp \left(-B_{n} \cdot Q_{n}^{2}\right),
$$

und wie man leicht nachprüfen kann, ist dann

$$
\left\langle Q_{n}^{2}\right\rangle=\frac{1}{2 B_{n}}=\frac{1}{2} \frac{h}{4 \pi^{2} v} \operatorname{ctgh} \frac{h v}{2 k T} \quad \text { für } \quad B_{n}>0,
$$

wobei $\left\langle Q_{n}{ }^{2}\right\rangle$ den Erwartungswert von $Q_{n}^{2}$ bedeutet. Durch Einsetzen von (10) und (7) in (1) folgt

$$
I_{i j}(s)=c_{i j} \int \ldots \int W\left(Q_{1}, \ldots, Q_{L}\right) \frac{\sin \left[s\left({ }^{(\mathrm{e})} r_{i j}+\sum_{n} c_{n} Q_{n}+\sum_{n} \sum_{m} k_{m n} Q_{m} Q_{n}\right)\right]}{s\left(^{(\mathrm{e})} r_{i j}+\sum_{n} c_{n} Q_{n}+\sum_{n} \sum_{m} k_{m n} Q_{m} Q_{n}\right)} \mathrm{d} Q_{1}, \ldots, \mathrm{d} Q_{L}
$$

5 F. Bцосн, Z. Phys. 74, 295 [1932]. 
mit $c_{i j}=$ const $\cdot f_{i} f_{j}$. Macht man für den Zähler in (12) die Näherung

$$
\sin \left(s r_{i j}\right) \approx \sin \left(s^{(\mathrm{e})} r_{i j}+s \sum_{n} c_{n} Q_{n}\right)+s \sum_{n} \sum_{m} k_{m n} Q_{m} Q_{n} \cos \left(s^{(\mathrm{e})} r_{i j}+s \sum_{n} c_{n} Q_{n}\right)
$$

und approximiert den Nenner $\operatorname{durch}{ }^{(e)} r_{i j}$ (siehe auch ${ }^{4}$ ), so kann man (12) in zwei Teilintegrale aufspalten:

$$
I_{i j}^{(1)}=c_{i j}(\operatorname{Im}) \prod_{n}\left(\frac{B_{n}}{\pi}\right)^{1 / 2}\left\{\frac{\exp \left(i s^{(\mathrm{e})} r_{i j}\right)}{s^{(\mathrm{e})} r_{i j}} \int_{-\infty}^{+\infty} \ldots \int_{-\infty}^{+\infty} \exp \left(-\sum_{n} B_{n} Q_{n}{ }^{2}+i s \sum_{n} c_{n} Q_{n}\right) \mathrm{d} Q_{1}, \ldots, \mathrm{d} Q_{L}\right.
$$

und

$$
\begin{aligned}
I_{i j}^{(2)}=c_{i j}(\mathrm{Re}) \prod_{n}\left(\frac{B_{n}}{\pi}\right)^{1 / 2}\left\{\frac{\exp \left(i s^{(\mathrm{e})} r_{i j}\right)}{{ }^{(\mathrm{e})} r_{i j}} \int_{-\infty}^{+\infty}\right. & \ldots \int_{-\infty}^{+\infty} \exp \left(-\sum_{n} B_{n} Q_{n}{ }^{2}+i s \sum_{n} c_{n} Q_{n}\right) \\
& \times \sum_{m} \sum_{n} k_{m n} Q_{m} Q_{n} \cdot \mathrm{d} Q_{1}, \ldots, \mathrm{d} Q_{L} .
\end{aligned}
$$

Darin bezeichnen (Re) und (Im) den Real- bzw. den Imaginärteil der Integrale. Die Integration von (14) liefert:

$$
I_{i j}^{(1)}=c_{i j} \exp \left(-\frac{s^{2}}{2} \sum_{n=1}^{L} \frac{c_{n}^{2}}{2 B_{n}}\right) \frac{\sin \left(s^{(\mathrm{e})} r_{i j}\right)}{s^{(\mathrm{e})} r_{i j}},
$$

während das Integral (15) - im Unterschied zu KARLE - gegeben ist durch

$$
I_{i j}^{(2)}=c_{i j}\left\{\sum_{n=1}^{L} k_{n n}\left(\frac{1}{2 B_{n}}-\frac{\left(s c_{n}\right)^{2}}{\left(2 B_{n}\right)^{2}}\right)-\sum_{\substack{n, m \\ n \neq m}} k_{m n} s^{2} \frac{c_{n} c_{m}}{4 B_{n} B_{m}}\right\} \times \exp \left(-\sum_{n} \frac{s^{2}}{2} \frac{c_{n}^{2}}{\left(2 B_{n}\right)^{2}}\right) \frac{\cos \left(s^{(\mathrm{e})} r_{i j}\right)}{s^{(\mathrm{e})} r_{i j}} .
$$

Nach Herleitung von KARLE-KaRLE ist

$$
\sum_{n=1}^{L} \frac{c_{n}^{2}}{2 B_{n}}=\left\langle\left(\sum_{n=1}^{L} c_{n} Q_{n}\right)^{2}\right\rangle=\left\langle\left(l_{i j}\right)^{2}\right\rangle,
$$

worin die $\left(l_{i j}\right)$ die Projektionen der Schwingungsamplituden auf die Verbindungslinie der Atome $i$ und $j$ in ihrer Gleichgewichtslage sind. $\left\langle\left(l_{i j}\right)^{2}\right\rangle$ stellt dementsprechend den Erwartungswert des Quadrats dieser Amplituden dar. Damit erhält (16) die bekannte Form

$$
I_{i j}^{(1)}=c_{i j} \exp \left(-\frac{s^{2}}{2}\left\langle l_{i j}{ }^{2}\right\rangle\right) \frac{\sin \left(s^{(\mathrm{e})} r_{i j}\right)}{s^{(\mathrm{e})} r_{i j}} .
$$

Für (17) folgt mit Hilfe von (11) und (18)

$$
I_{i j}^{(2)}=c_{i j} \frac{\cos \left(s^{(\mathrm{e})} r_{i j}\right)}{{ }^{(\mathrm{e})} r_{i j}} \exp \left(-\frac{s^{2}}{2}\left\langle l_{i j}{ }^{2}\right\rangle\right)\left(\sum_{n} k_{n n}\left\langle Q_{n}{ }^{2}\right\rangle-s^{2} \sum_{m} \sum_{n} k_{m n} c_{m}\left\langle Q_{m}{ }^{2}\right\rangle c_{n}\left\langle Q_{n}{ }^{2}\right\rangle\right),
$$

und mit Hilfe von (9) und (18)

$$
I_{i j}^{(2)}=c_{i j} \frac{\cos \left(s^{(\mathrm{e})} r_{i j}\right)}{{ }^{(\mathrm{e})} r_{i j}} \exp \left(-\frac{s^{2}}{2}\left\langle l_{i j}{ }^{2}\right\rangle\right) \frac{1}{2^{(\mathrm{e})} r_{i j}}\left(s^{2}\left\langle l_{i j}{ }^{2}\right\rangle^{2}-\left\langle l_{i j}{ }^{2}\right\rangle\right) .
$$

Die gesamte erweiterte Intensitätsfunktion bei Berechnung der Schwingungen in der harmonischen Näherung folgt aus (19) und (21) zu

$$
I_{i j}(s)=c_{i j} \exp \left(-\frac{s^{2}}{2}\left\langle l_{i j}{ }^{2}\right\rangle\right)\left\{\frac{\sin \left(s^{(\mathrm{e})} r_{i j}\right)}{s^{(\mathrm{e})} r_{i j}}-\frac{\left\langle l_{i j}{ }^{2}\right\rangle}{2}\left(1-s^{2}\left\langle l_{i j}{ }^{2}\right\rangle\right) \frac{\cos \left({ }^{(\mathrm{e})} r_{i j}\right)}{{ }^{(\mathrm{e})} r_{i j}{ }^{2}}\right\} .
$$

Indem wir der Vorschrift von DeBye ${ }^{4}$ folgen, bilden wir mit $I_{i j}(s)$ aus (22) die Fourier-Transformation und erhalten so die Radialverteilungsfunktion $D\left(r_{i j}\right)$

$$
D\left(r_{i j}\right)=\frac{\pi}{2} c_{i j} \frac{P\left(r_{i j}\right)}{r_{i j}}=\int_{0}^{\infty} s \cdot I_{i j}(s) \cdot \sin \left(s r_{i j}\right) \mathrm{d} s .
$$


Mit Hilfe von (22) und nach einigen elementaren Umformungen des Integranden ergibt sich schließlich

$$
\begin{aligned}
\frac{P\left(r_{i j}\right)}{r_{i j}} & =\frac{2}{\pi} \int_{0}^{\infty} \exp \left(-\frac{s^{2}}{2}\left\langle l_{i j}{ }^{2}\right\rangle\right) \frac{1}{2^{(\mathrm{e})} r_{i j}}\left[\cos \left(s\left[{ }^{(\mathrm{e})} r_{i j}-r_{i j}\right]\right)-\cos \left(s\left[{ }^{(\mathrm{e})} r_{i j}+r_{i j}\right]\right)\right] \mathrm{d} s \\
& -\frac{\left\langle l_{i j}{ }^{2}\right\rangle}{\pi}\left\{\int_{0}^{\infty} \exp \left(-\frac{s^{2}}{2}\left\langle l_{i j}{ }^{2}\right\rangle\right) \frac{s}{2^{(\mathrm{e})} r_{i j}{ }^{2}}\left[\sin \left(s\left[r_{i j}-{ }^{(\mathrm{e})} r_{i j}\right]\right)+\sin \left(s\left[r_{i j}+{ }^{(\mathrm{e})} r_{i j}\right]\right)\right] \mathrm{d} s\right. \\
& \left.-\int_{0}^{\infty} \exp \left(-\frac{s^{2}}{2}\left\langle l_{i j}{ }^{2}\right\rangle\right) \frac{s^{3}\left\langle l_{i j}{ }^{2}\right\rangle}{2^{(\mathrm{e})} r_{i j}{ }^{2}}\left[\sin \left(s\left[r_{i j}-{ }^{(\mathrm{e})} r_{i j}\right]\right)+\sin \left(s\left[r_{i j}+{ }^{(\mathrm{e})} r_{i j}\right]\right)\right] \mathrm{d} s\right\} \\
& =P_{1}-\left(P_{2}-P_{3}\right) .
\end{aligned}
$$

Das Integral $P_{1}$ ist das gleiche wie bei KARLE-KARLE, während in der erwähnten Arbeit $P_{2}$ und $P_{3}$ vernachlässigt werden:

$$
P_{1}=\frac{1}{{ }^{(\mathrm{e})} r_{i j}\left(2 \pi\left\langle l_{i j}{ }^{2}\right\rangle\right)^{1 / 2}}\left[\exp \left(-\frac{\left(^{(\mathrm{e})} r_{i j}-r_{i j}\right)^{2}}{2\left\langle l_{i j}{ }^{2}\right\rangle}\right)-\exp \left(-\frac{\left.{ }^{(\mathrm{e})} r_{i j}+r_{\mathrm{ij}}\right)^{2}}{2\left\langle l_{i j}{ }^{2}\right\rangle}\right)\right] .
$$

Für die Integrale $P_{2}$ und $P_{3}$ findet man die Ausdrücke ${ }^{6}$ :

$$
\begin{gathered}
P_{2}=\frac{1}{2^{(\mathrm{e})} r_{i j}{ }^{2}\left(2 \pi\left\langle l_{i j}{ }^{2}\right\rangle\right)^{1 / 2}}\left[\left(r_{i j}-{ }^{(\mathrm{e})} r_{i j}\right) \exp \left(-\frac{\left(r_{i j}-{ }^{(\mathrm{e})} r_{i j}\right)^{2}}{2\left\langle l_{i j}{ }^{2}\right\rangle}\right)+\left(r_{i j}+{ }^{(\mathrm{e})} r_{i j}\right) \exp \left(-\frac{\left(r_{i j}+{ }^{(\mathrm{e})} r_{i j}\right)^{2}}{2\left\langle l_{i j}{ }^{2}\right\rangle}\right)\right], \\
P_{3}=-\frac{1}{2^{(\mathrm{e})} r_{i j}{ }^{2}\left(2 \pi\left\langle l_{i j}{ }^{2}\right\rangle\right)^{1 / 2}}\left[\frac{\left(r_{i j}-{ }^{(\mathrm{e})} r_{i j}\right)^{3}}{\left\langle l_{i j}{ }^{2}\right\rangle} \exp \left(-\frac{\left(r_{i j}-{ }^{(\mathrm{e})} r_{i j}\right)^{2}}{2\left\langle l_{i j}{ }^{2}\right\rangle}\right)\left(1-3 \frac{\left\langle l_{i j}{ }^{2}\right\rangle}{\left(r_{i j}-{ }^{(\mathrm{e})} r_{i j}\right)^{2}}\right)\right. \\
\left.+\frac{\left(r_{i j}+{ }^{\left({ }^{2}\right)} r_{i j}\right)^{3}}{\left\langle l_{i j}\right\rangle} \exp \left(-\frac{\left(r_{i j}+{ }^{\left({ }^{(\mathrm{e}}\right)} r_{i j}\right)^{2}}{2\left\langle l_{i j}{ }^{2}\right\rangle}\right)\left(1-3 \frac{\left\langle l_{i j} j^{2}\right\rangle}{\left(r_{i j}+{ }^{(\mathrm{e})} r_{i j}{ }^{2}\right.}\right)\right] .
\end{gathered}
$$

Die Zusammenfassung von (25), (26) und (27) ergibt folgenden Ausdruck für die Radialverteilungsfunktion:

$$
\begin{aligned}
& \frac{P\left(r_{i j}\right)}{r_{i j}}=\left[\exp \left(-\frac{\left.{ }^{(\mathrm{e})} r_{i j}-r_{i j}\right)^{2}}{2\left\langle l_{i j}\right\rangle}\right)\left(1+\frac{\left(r_{i j}-{ }^{(\mathrm{e})} r_{i j}\right)}{{ }^{(\mathrm{e})} r_{i j}}-\frac{\left({ }^{2} i j-{ }^{(\mathrm{e})} r_{i j}\right)^{3}}{2^{(\mathrm{e})} r_{i j}\left\langle l_{i j}{ }^{2}\right\rangle}\right)\right. \\
& \left.-\exp \left(-\frac{\left.{ }^{(\mathrm{e})} r_{i j}+r_{i j}\right)^{2}}{2\left\langle l_{i j}{ }^{2}\right\rangle}\right)\left(1-\frac{\left(r_{i j}+{ }^{\left({ }^{(e)}\right.} r_{i j},\right.}{{ }^{\left({ }^{e}\right)} r_{i j}}+\frac{\left(r_{i j}+{ }^{(\mathrm{e})} r_{i j}\right)^{3}}{2^{(\mathrm{e})} r_{i j}\left\langle l_{i j}{ }^{2}\right\rangle}\right)\right] \times \frac{1}{{ }^{(\mathrm{e})} r_{i j}\left(2 \pi\left\langle l_{i j}{ }^{2}\right\rangle\right)^{1 / 2}} \cdot
\end{aligned}
$$

Die Rechnung an einigen Beispielen zeigt, daß für die praktische Auswertung der Term mit der negativen Exponentialfunktion vernachlässigt werden kann.

\section{Diskussion}

Die Zusatzterme, die in der Intensitätsfunktion (22) und der Radialverteilungsfunktion (28) neu hinzugekommen sind, resultieren aus dem quadratischen Term in der Entwicklung (5). Demzufolge muß eine genauere Untersuchung all dieser Terme eine Aussage über die physikalische Bedeutung der neuen Funktionen (22) und (28) liefern.

a) Um eine Vorstellung von der Größe des Unterschiedes zwischen den beiden Intensitätsfunktionen (19) und (22) zu bekommen, haben wir für eine Reihe von bekannten Molekülen Modellrechnungen durchgeführt und dabei folgende charakteristischen Unterschiede festgestellt. Für kleine $s$-Werte sind die relativen Änderungen der neuen Intensitätsfunktion gegenüber der alten von kleinerer Ordnung als für große $s$-Werte. Für große $s$-Werte ist die Dämpfung der Intensität auf Grund der Zusatzterme kleiner als bei der alten Intensitätsfunktion. Eine Verschiebung der Nullstellen in eine bestimmte Richtung ist nicht immer feststellbar.

Eine Gegenüberstellung der alten und der neuen Intensitätsfunktion am Beispiel des $\mathrm{CCl}_{4}$ zeigt Abb. 1 . Die Zusammenfassung der Strukturparameter für $\mathrm{CCl}_{4}$ findet sich in Tab. 1 .

Abb. 2 zeigt, daß die erweiterte Radialverteilungsfunktion (28) eine Verschiebung des Maximums zu größeren Abständen bringt. Deutliche $\mathrm{Ab}$ weichungen von der Gestalt der Gaussschen Verteilungsfunktion sind in der Nähe von $r_{\mathrm{e}}$ und im Bereich $\left|r_{i j}-r_{\mathrm{e}}\right|>l_{i j}$ zu beobachten. Die Funktion

${ }^{6}$ Siehe z. B. Integraltafeln von Gröbner und Hofreiter, Springer-Verlag, Wien 1961. 


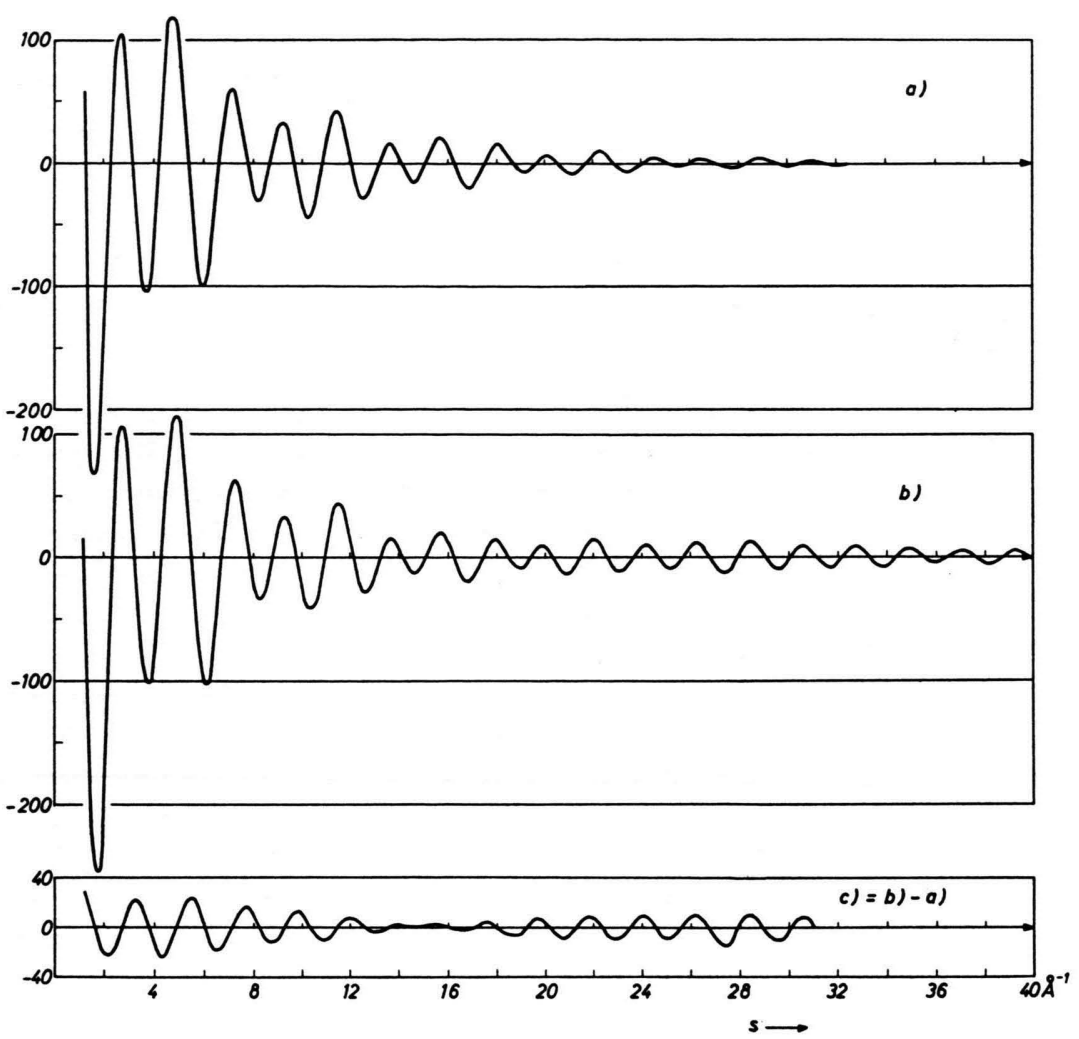

Abb. 1. Theoretische Intensitätsfunktionen für Tetrachlorkohlenstoff.

a) Berechnet nach Gl. (19) mit: $r_{\mathrm{CCl}}=1,769 \AA, \quad r_{\mathrm{ClCl}}=2,884 \AA$, $l_{\mathrm{CCl}}=0,065 \AA, \quad l_{\mathrm{ClCl}}=0,67 \AA$.

b) Berechnet nach $\mathrm{Gl}$. (22) mit den nach Gl. (33) korrigierten Abständen : $\quad r_{\mathrm{CCl}}=1,766_{5}$ und

c) Differenzkurve. $r_{\mathrm{ClCl}}=2,882_{5} \AA$.

Eine Zusammenstellung von Strukturparametern, korrigierten Abständen und Abweichungen vom regulären Tetraeder des $\mathrm{CCl}_{4}$ findet man in Tab. 1.

\begin{tabular}{|c|c|c|c|}
\hline & BARTELL et al..$^{\mathbf{a}}$ & Morino et al. b & HaAse-Zeil $^{c}$ \\
\hline $\begin{array}{l}r_{\mathrm{CCl}} \\
r_{\mathrm{ClCl}} \\
l_{\mathrm{CCl}} \\
l_{\mathrm{ClCl}}\end{array}$ & $\begin{array}{l}1,769 \pm 0,005 \\
2,887 \pm 0,004 \\
0,060 \pm 0,005 \\
0,068 \pm 0,003\end{array}$ & $\begin{array}{l}1,767 \pm 0,003 \\
2,888 \pm 0,003 \\
0,051 \pm 0,002 \\
0,069 \pm 0,001\end{array}$ & \begin{tabular}{l|}
$1,769 \pm 0,002$ \\
$2,884 \pm 0,0005$ \\
$0,065 \pm 0,002$ \\
$0,067 \pm 0,0005$
\end{tabular} \\
\hline \multicolumn{4}{|c|}{ Abweichungen $\varepsilon$ vom Tetraeder ${ }^{d}$} \\
\hline$\varepsilon$ & $0,001_{7}$ & $-0,0025$ & $0,004_{7}$ \\
\hline \multicolumn{4}{|c|}{ korrigierte Abstände ${ }^{e}$} \\
\hline $\begin{array}{l}r_{\mathrm{CCl}} \\
r_{\mathrm{ClCl}}\end{array}$ & $\begin{array}{l}1,767 \\
2,885_{3}\end{array}$ & $\begin{array}{l}1,765_{6} \\
2,886_{3}\end{array}$ & $\begin{array}{l}1,766_{5} \\
2,882_{5}\end{array}$ \\
\hline \multicolumn{4}{|c|}{ Abweichungen $\varepsilon_{\text {korr }}$ vom Tetraeder ${ }^{f}$} \\
\hline$\varepsilon_{\text {korr }}$ & 0,0002 & $-0,0031$ & 0,0013 \\
\hline
\end{tabular}

a L. S. Bartell, L. O. Brockway u. R. H. Schwendemann, J. Chem. Phys. 23,1854 [1955].

b Y. Morino, Y. Nakamura u. T. Iijima, J. Chem. Phys. 32, 643 [1960].

c J. HaAse u. W. Zeir, Z. Phys. Chem. Frankfurt 45, 202 [1965].

${ }^{d} \varepsilon=\left(1,63299 \cdot r_{\mathrm{CCl}}-r_{\mathrm{ClCl}}\right)$.

e Die Abstände sind gemäß Gl. (33) korrigiert worden.

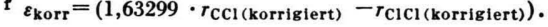

Tab. 1 (zu Abb. 1), alle Größen in $\AA$.

selbst ist nicht symmetrisch bezüglich $r_{\mathrm{e}}$ und zeigt ähnliche Asymmetrien, wie sie durch Anharmonizitäten im Potential verursacht werden. Das „non-negativity-Kriterium“ von KARLE-KarLe gilt im Rahmen

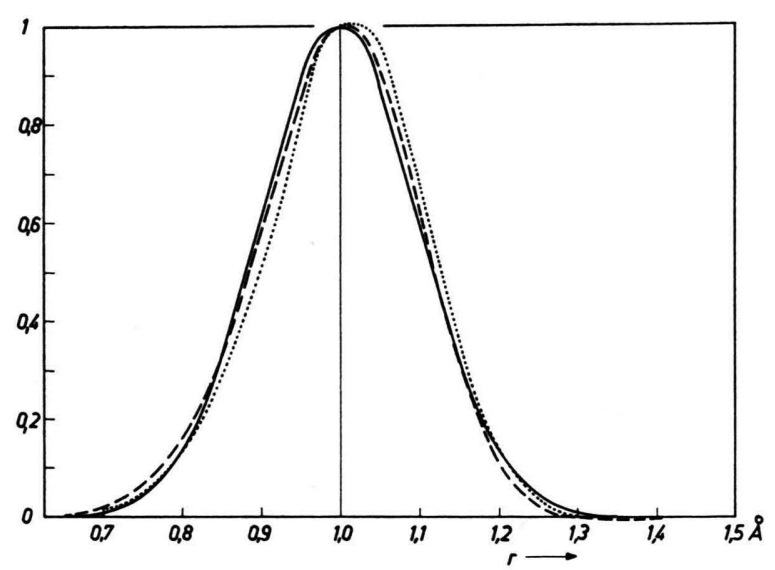

Abb. 2. Gausssche Radialverteilungsfunktion (- $(-)$ Verteilungsfunktion mit kinematischer Asymmetrie (- - - ), Verteilungsfunktion eines anharmonischen Oszillators $(\cdots \cdots)$ mit $a=3 \AA^{-1}$ (siehe z. B. ${ }^{8}$ ); alle drei Kurven sind für $b=0$, $r_{\mathrm{e}}=1 \AA$ und $l_{i j}=0,10 \AA$ berechnet worden.

der Näherung, die im Abschnitt 1. gemacht wurde und die auf die neuen Ergebnisse führte, nicht streng. Zum Aufsuchen eines optimalen Restuntergrundes ist es aber nach wie vor - als bisher einzige anwendbare Richtlinie - gültig. 
Die Größe $r_{\mathrm{e}}$ können wir entweder aus der Anpassung der Gl. (28) an die experimentelle Radialverteilungsfunktion entnehmen oder über eine Relation zwischen $r_{\mathrm{e}}$ und $r_{\max }$ bestimmen. Dabei ist $r_{\max }$ als Nullstelle von

$$
\left.\frac{\mathrm{d}}{\mathrm{d} r_{i j}}\left(\frac{P\left(r_{i j}\right)}{r_{i j}}\right)\right|_{r_{i j}=r_{\max }}=0
$$

definiert. Einsetzen von (28) in (29) führt dann auf die Gleichung

$\Delta r^{4}-5\left\langle l_{i j}{ }^{2}\right\rangle \Delta r^{2}-2^{(\mathrm{e})} r_{i j}\left\langle l_{i j}{ }^{2}\right\rangle \Delta r+2\left\langle l_{i j}{ }^{2}\right\rangle=0$,

wobei $\Delta r=r_{\max }-{ }^{(\mathrm{e})} r_{i j}$ gesetzt wurde. Die Werte dieser Korrekturen $\Delta r$ für verschiedene $l_{i j}$ und ${ }^{(\mathrm{e})} r_{i j}$ entnimmt man der Kurvenschar der Abb. 3. Wie man leicht nachprüft, kann man $\Delta r$ in sehr guter Nähe-

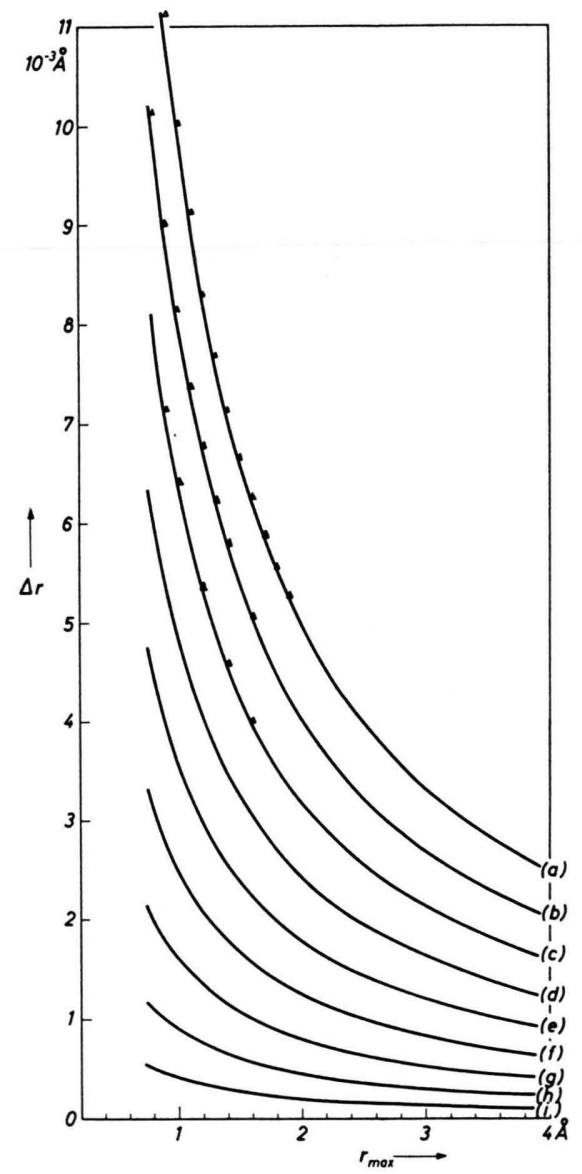

Abb. 3. $\Delta r$ sind die Lösungen von Gl. (30). Kurvenparameter $l_{i j}=0,10 \AA$ (a), $0,09 \AA(\mathrm{b}), 0,08 \AA$ (c), $0,07 \AA$ (d), $0,06 \AA$ (e), $0,05 \AA$ (f), $0,04 \AA$ (g), $0,03 \AA$ (h), $0,02 \AA$ (i). Die mit $\triangle$ markierten Punkte sind aus Gl. (33) berechnet. Die Näherung (31) ist also sehr gut. rung auch aus

$-5\left\langle l_{i j}{ }^{2}\right\rangle \Delta r^{2}-2^{(\mathrm{e})} r_{i j}\left\langle l_{i j}{ }^{2}\right\rangle \Delta r+2\left\langle l_{i j}{ }^{2}\right\rangle=0$

berechnen; dies ergibt

$$
\Delta r=\frac{1}{5}\left(-{ }^{(\mathrm{e})} r_{i j}+\left(10\left\langle l_{i j}{ }^{2}\right\rangle+{ }^{(\mathrm{e})} \boldsymbol{r}_{i j}{ }^{2}\right)^{1 / 2}\right)
$$

und, wenn man nach ${ }^{(\mathrm{e})} r_{i j}$ auflöst und die dabei auftretende Wurzel entwickelt,

$$
{ }^{(\mathrm{e})} \boldsymbol{r}_{i j}=r_{\max }-\left\langle l_{i j}{ }^{2}\right\rangle / r_{\max } \cdot
$$

Prinzipiell könnte man (30) auch so auswerten, daß man ${ }^{(\mathrm{e})} \boldsymbol{r}_{i j}$ aus $\Delta \boldsymbol{r}=\boldsymbol{r}_{\min }-{ }^{(\mathrm{e})} \boldsymbol{r}_{i j}$ berechnet. Dies empfiehlt sich aber nicht, da wegen der Untergrundungenauigkeit die Lage von $r_{\min }$ in der experimentellen Radialverteilungsfunktion sehr ungenau sein kann. Man kann aber umgekehrt die Lage von $r_{\min }$ aus (31) berechnen, nachdem man vorher ${ }^{(\mathrm{e})} \boldsymbol{r}_{i j}$ aus der Lage von $r_{\max }$ berechnet hat, und hat somit ein weiteres Kriterium für die Güte des Untergrundes.

b) Zur Interpretation der oben erwähnten Asymmetrien der Radialverteilungsfunktion, welche auf Grund der hier verwendeten harmonischen Näherung nicht auf Anharmonizitäten des Potentials zurückgeführt werden können, führen wir folgende geometrische Betrachtung durch.

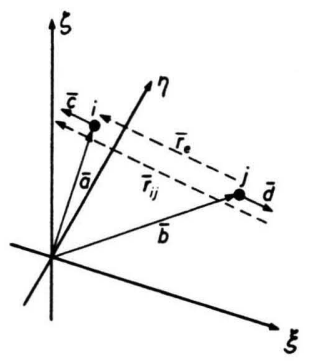

Abb. 4. Zweiatomiges Molekül; $\bar{c}$ und $\bar{d}$ bezeichnen die momentanen Vektoren der Schwingungsamplituden von $i$ bzw. $j$.

a) Zweiatomige Moleküle (Abb.4): Die Schwingungen aus der Gleichgewichtslage erfolgen in Richtung der Kernverbindungslinie. Mit den Bezeichnungen der Abb. 4 gilt für den momentanen $\mathrm{Ab}$ stand $\bar{r}_{i j}$ unter Annahme von harmonischen Schwingungen zwischen Atom $i$ und $j$ :

$$
\begin{aligned}
\overline{r_{i j}} & =\bar{a}+\bar{c}-(\bar{b}+\bar{d}) \\
& =(\bar{a}-\bar{b})+(\bar{c}-\bar{d}) \\
& \left.=\overline{r_{\mathrm{e}}}+\overline{(c}-\bar{d}\right)
\end{aligned}
$$

und in den Koordinaten $\xi, \eta, \zeta$

$$
\begin{aligned}
\bar{r}_{i j} & =\left(\xi_{i j}, \eta_{i j}, \zeta_{i j}\right)+\left(\xi^{i j}, \eta^{i j}, \zeta^{i j}\right) \\
& =\left(\xi_{i j}+\xi^{i j}, \eta_{i j}+\eta^{i j}, \zeta_{i j}+\zeta^{i j}\right) .
\end{aligned}
$$


Für den Betrag $\left|\overline{r_{i j}}\right|$ gilt

$$
\begin{aligned}
\left|\bar{r}_{i j}\right|=\left|\bar{r}_{\mathrm{e}}+(\bar{c}-\bar{d})\right| & \leqq\left|\bar{r}_{\mathrm{e}}\right|+|\bar{c}-\bar{d}|= \\
& =\bar{r}_{\mathrm{e}}+\frac{\overline{r_{\mathrm{e}}}}{\left|\mathrm{re}_{\mathrm{e}}\right|} \cdot(\bar{c}-\bar{d}) .
\end{aligned}
$$

Wegen der linearen Abhängigkeit von $\overline{r_{\mathrm{e}}}$ und $(\bar{c}-\bar{d})$ steht hier das Gleichheitszeichen. In Koordinatenschreibweise erhält (36) die Form

$$
\left|\bar{r}_{i j}\right|=\left|\bar{r}_{\mathrm{e}}\right|+\frac{1}{\left|\bar{r}_{\mathrm{e}}\right|}\left(\xi_{i j} \xi^{i j}+\eta_{i j} \eta^{i j}+\zeta_{i j} \zeta^{i j}\right) .
$$

Ein Vergleich mit (5) zeigt, daß im Falle der zweiatomigen Molekeln der quadratische Term in (5) verschwindet. Damit erfahren hier die Abstandsänderungen der Atome $i$ und $j$ bei Bewegungen im harmonischen Potential keine kinematische Asymmetrie und die Zusatzterme in (22) und (28) entfallen.

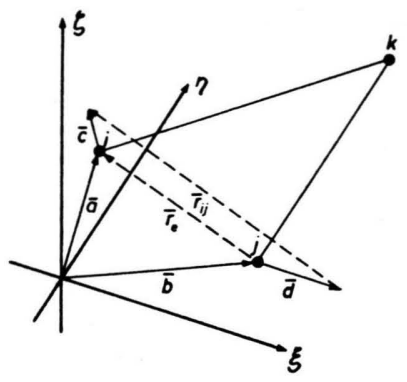

Abb. 5. Mehratomiges Molekül; ${ }^{-}$e parallel ${ }_{r i j},|\bar{c}|,|\bar{d}|$ beliebig.

B) Mehratomige Moleküle (Abb. 5): Für den Fall, daß in einem Molekül ein Atompaar $(i, j)$ harmonische Schwingungen ausführt derart, $\mathrm{da} \beta$ in jedem Augenblick der Abstand $\overline{r_{i j}}$ parallel zu (e) $\overline{r_{i j}}$ ist, gelten die gleichen Betrachtungen und die gleichen Folgerungen wie unter $\alpha$ ). Für alle anderen Schwingungstypen gelten die in Abschnitt 1 durchgeführten Herleitungen.

c) Für den physikalischen Hintergrund der Betrachtungen und Resultate unter $2 \mathrm{~b}$ und die Berechnungen in 1 kann man vom Standpunkt der Punktmechanik folgende anschauliche Interpretation für die Bewegungsvorgänge im Molekül geben, durch die die Abstandsänderungen bezüglich der Gleichgewichtslage unsymmetrisch werden (Abb.6). Die Abbildung zeigt am Beispiel eines $\mathrm{XY}_{2}$-Moleküls (wobei das Massenverhältnis X : Y mit $1: 2$ gewählt wurde), wie bei einer Normalschwingung kinematische Asymmetrien zustande kommen. Die auf

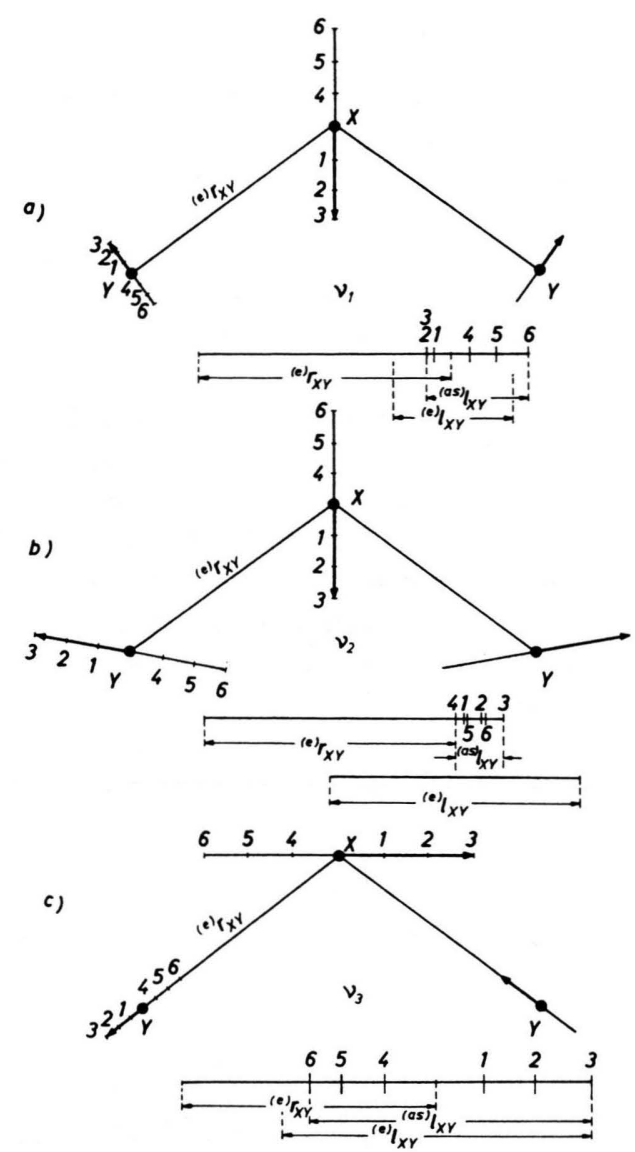

Abb. 6. Modell eines $\mathrm{XY}_{2}$-Moleküls zur Beschreibung der kinematischen Asymmetrie. Erläuterungen siehe Text.

den Vektoren in äquidistanten Schritten markierten Punkte $1, \ldots, 6$ stellen die einander zugeordneten Atomlagen während des gleichphasigen Bewegungsablaufes der drei Atome dar. Die momentanen Atomabstände $(1,1), \ldots,(6,6)$ sind nicht symmetrisch um die Gleichgewichtslage ${ }^{(\mathrm{e})} r_{\mathrm{XY}}$ verteilt, sondern zeigen eine asymmetrische Verteilung um ${ }^{(e)} r_{X Y} \cdot$ Unter den jeweiligen Normalschwingungsbildern ist dieser Sachverhalt gesondert dargestellt. Man erkennt, daß die Verteilung der Abstände um ${ }^{(e)} r_{X Y}$ erstens asymmetrisch und zweitens nicht äquidistant ist. Zum Vergleich mit der so erhaltenen Schwingungsamplitude ${ }^{(\mathrm{as})} l_{\mathrm{XY}}$ ist die gebräuchliche Schwingungsamplitude ${ }^{(\mathrm{e})} l_{\mathrm{XY}}$, die symmetrisch bezüglich ${ }^{(\mathrm{e})} r_{\mathrm{XY}}$ ist, eingezeichnet. Unter den drei Grundschwingungen $v_{1}, v_{2}, v_{3}$ läßt die unter b) dargestellte Verteilung die kinematische Asymmetrie besonders klar erkennen. 


\section{Bemerkungen}

Zur Verbesserung der neuen Intensitätsfunktion muß die Wahrscheinlichkeitsfunktion $W\left(Q_{1}, \ldots, Q_{L}\right)$ [siehe Gl. (10) ] entsprechend der Anharmonizität des Potentials abgeändert werden. Nähert man es z. B. durch ein Morse-Potential ${ }^{7}$ an und verwendet die hierzu gehörende, bei Bartell ${ }^{8}$ angegebene Wahrscheinlichkeitsfunktion, so kann man mit Hilfe eines ähnlichen Lösungsweges wie unter 1 . die Intensitätsfunktion berechnen. Berechnungen in dieser Richtung sind in Bearbeitung und es dürfte möglich sein, bei Verwendung der Lösungsmethode von 1 . einen geschlossenen Ausdruck für $I_{i j}(s)$ zu erhalten, bei dem sich die Anharmonizität des Potentials nicht mehr durch eine nur stückweise geschlossen darstellbare Phasenverschiebung $\Phi(s)$, wie sie Bartell berechnete, ausdrückt.

Weiterhin müssen die vernachlässigten Terme $\left(\xi^{i j}\right)^{2},\left(\eta^{i j}\right)^{2},\left(\zeta^{i j}\right)^{2}$ in (5) bei einer genaueren Be- rechnung der Intensitätsfunktion berücksichtigt werden. Diese Terme sind für das Auftreten des von Morino ${ }^{9}$ berechneten „shrinkage“-Effektes verantwortlich. Denn führt man eine Abbildung des speziellen Koordinatensystems $x, y, z$ von Morino auf das allgemeine Koordinatensystem $\xi, \eta, \zeta$ durch, so treten die $\left(\xi^{i i}\right)^{2}$ usw. an die Stelle der $(\Delta x)^{2},(\Delta y)^{2}$, $(\Delta z)^{2}$ bei Morino. Auch hierüber sind genauere Untersuchungen in Vorbereitung. Was wir bisher aussagen können ist, daß bei Anwendung der Korrekturen $\Delta r$ auf die Abstände $r_{\max }$ (z. B. ${ }^{9-11}$ ) die Diskrepanz zwischen dem berechneten "shrinkage“-Effekt $\delta r_{\text {ber }}$ und dem beobachteten "shrinkage“-Effekt $\delta r_{\exp }$ noch vergrößert wird. Eine Übersicht hierüber gibt Tab. 2.

Herrn Dipl.-Phys. Dr. W. HürtNer danken wir für wertvolle Diskussionen über die Interpretation der $\mathrm{Zu}$ satzterme in Gl. (22) und (28) mit Hilfe von kinematischer Asymmetrie.

\begin{tabular}{|c|c|c|c|c|c|c|c|}
\hline \multicolumn{8}{|c|}{ a) Allen $\mathrm{H}_{2} \mathrm{C}=\mathrm{C}=\mathrm{CH}_{2}$} \\
\hline Abstand & $r_{\max } 11$ & $l_{i j^{11}}$ & $r_{\text {korr }} *$ & $\delta_{\text {beob }^{11}}$ & $\delta_{\mathrm{g}(\text { beob) }}{ }^{9}$ & $\delta_{\text {ber }}{ }^{9}$ & $\delta_{\text {exp, korr }} * *$ \\
\hline $\begin{array}{l}\mathrm{C} \equiv \mathrm{C} \\
\mathrm{C} \ldots \mathrm{C}\end{array}$ & $\begin{array}{l}1,3116 \\
2,6173\end{array}$ & $\begin{array}{l}0,039 \\
0,051_{9}\end{array}$ & $\begin{array}{l}1,3104 \\
2,6183\end{array}$ & $\overline{0,005_{9}}$ & $\begin{array}{c}- \\
0,010_{7} \ldots 0,007_{9}\end{array}$ & $0, \overline{010}$ & $0, \overline{004} 5$ \\
\hline \multicolumn{8}{|c|}{ b) Dimethyldiacetylen $\mathrm{H}_{3} \mathrm{C}-\mathrm{C} \equiv \mathrm{C}-\mathrm{C} \equiv \mathrm{C}-\mathrm{CH}_{3}$} \\
\hline $\begin{array}{l}\mathrm{C}_{1}-\mathrm{C}_{2} \\
\mathrm{C}_{2} \equiv \mathrm{C}_{3} \\
\mathrm{C}_{3}-\mathrm{C}_{4} \\
\mathrm{C}_{1} \ldots \mathrm{C}_{3} \\
\mathrm{C}_{1} \ldots \mathrm{C}_{4} \\
\mathrm{C}_{1} \ldots \mathrm{C}_{5} \\
\mathrm{C}_{1} \ldots \mathrm{C}_{6} \\
\mathrm{C}_{2} \ldots \mathrm{C}_{4} \\
\mathrm{C}_{2} \ldots \mathrm{C}_{5}\end{array}$ & $\begin{array}{l}1,452 \\
1,210 \\
1,379 \\
2,661 \\
4,026 \\
5,228 \\
6,661 \\
2,590 \\
3,778\end{array}$ & $\begin{array}{l}0,061 \\
0,040 \\
0,061 \\
0,066 \\
0,060 \\
0,072 \\
0,080 \\
0,050 \\
0,049\end{array}$ & $\begin{array}{l}1,449_{5} \\
1,208_{7} \\
1,376_{4} \\
2,659 \\
4,025_{1} \\
5,226_{9} \\
6,660 \\
2,589_{9} \\
3,777_{4}\end{array}$ & $\begin{array}{c}\overline{-} \\
\overline{-} \\
0,001 \\
0,015 \\
0,023 \\
0,042 \\
0,001 \\
0,021\end{array}$ & $\begin{array}{c}- \\
\overline{-} \\
0,003 \\
0,021 \\
0,030 \\
0,052 \\
0,002 \\
0,026\end{array}$ & $\begin{array}{c}\overline{-} \\
\overline{-} \\
0,012 \\
0,027 \\
0,050 \\
0,073 \\
0,013 \\
0,031\end{array}$ & $\begin{array}{c}\overline{-} \\
\overline{-} \\
-0,001_{2} \\
0,009_{5} \\
0,016_{4} \\
0,032_{8} \\
-0,004 \\
0,016_{4}\end{array}$ \\
\hline
\end{tabular}

* Errechnet aus Gl. (33) dieser Arbeit aus $r_{\max }$ und $l_{i j}$.

** Errechnet aus $r_{\text {korr }}$. $\delta_{\text {beob }}$ bzw. $\delta_{\text {exp }}$ bezeichnen den experimentellen „shrinkage“.Effekt.

Tab. 2. Vergleich des beobachteten und berechneten Bastians EN-Morino-shrinkage-Effekts am Allen und Dimethyldiacetylen (alle Größen in $\AA$ ). Man beachte, daß alle Daten in der Literatur ohne Fehlerangaben sind.

7 P. M. Morse, Phys. Rev. 34, 57 [1929].

8 L. S. Bartell, J. Chem. Phys. 23, 1219 [1955]. - K. KUChitsu u. L. S. Bartell, J. Chem. Phys. 35, 1945 [1961].

9 Y. Morino, J. Nakamura u. P. W. Moore, J. Chem. Phys. 36, 1050 [1962].
10 A. Almenningen, O. Bastiansen u. T. Munthe-Katas, Acta Chem. Scand. 10, 261 [1956].

11 A. Almenningen, O. Bastiansen u. M. Traetteberg, Acta Chem. Scand. 13, 1699 [1959]. 